\title{
All-trans-retinoic acid modulates TGF- $\beta$-induced apoptosis, proliferation, migration and extracellular matrix synthesis of conjunctival fibroblasts by inhibiting PI3K/AKT signaling
}

\author{
LINGLING LIANG $^{1,2}$, XIAOMEI WANG ${ }^{2}$, YAJUAN ZHENG ${ }^{1}$ and YANG LIU ${ }^{3}$ \\ ${ }^{1}$ Department of Ophthalmology, The Second Hospital of Jilin University, Jilin University, Changchun, \\ Jilin 130041; ${ }^{2}$ Department of Ophthalmology, The First Hospital of Jilin University, Jilin University, \\ Changchun, Jilin 130021; ${ }^{3}$ Department of Ophthalmology, The Fifth Affiliated Hospital, \\ Sun Yet-Sen University, Zhuhai, Guangdong 519000, P.R. China
}

Received November 27, 2018; Accepted June 24, 2019

DOI: $10.3892 / \mathrm{mmr} .2019 .10507$

\begin{abstract}
Conjunctival fiber generation is implicated in a wide spectrum of ocular diseases. Conjunctival wound healing is characterized by inflammation followed by re-epithelialization, synthesis of new extracellular matrix (ECM), wound contraction and subconjunctival scar formation. The primary cause for the failure of glaucoma filtration surgery results from the excessive scarring of the filtering bleb. All-trans-retinoic acid (ATRA), a derivative of vitamin A, is a potent regulator of ECM synthesis, growth and differentiation. Following a previous study, which revealed that ATRA could inhibit transforming growth factor- $\beta$-induced human conjunctival fibroblast (HConF)-mediated collagen gel contraction, the present study aimed to investigate the effects of ATRA on HConF migration, apoptosis, proliferation and ECM synthesis. To achieve this, the present study used Transwell migration, wound healing and Cell Counting Kit- 8 assays, flow cytometry and western blot analysis. In addition, the present study aimed to elucidate the mechanism of ATRA in mediating resistance to conjunctival scar formation. ATRA treatment resulted in an increased level of HConF apoptosis, reduced proliferation and migration, decreased collagen I and fibronectin expression, and decreased phosphorylation of PI3K and AKT. Thus, the present study showed a role for ATRA in inhibiting HConF migration, proliferation and ECM synthesis, and in promoting
\end{abstract}

Correspondence to: Professor Yajuan Zheng, Department of Ophthalmology, The Second Hospital of Jilin University, Jilin University, 218 Ziqiang Road, Nanguan, Changchun, Jilin 130041, P.R. China

E-mail: zhengyajuan124@126.com

Professor Yang Liu, Department of Ophthalmology, The Fifth Affiliated Hospital, Sun Yet-Sen University, 52 Meihuadong Road, Zhuhai, Guangdong 519000, P.R. China

E-mail: yangliu_manu@163.com

Key words: all-trans-retinoic acid, transforming growth factor- $\beta$, conjunctival fibroblasts, PI3K/AKT
HConF apoptosis through the inhibition of the PI3K/AKT signaling pathway.

\section{Introduction}

Glaucoma is the most common cause of irreversible blindness worldwide, accounting for $\sim 15 \%$ of all cases and $\geq 500,000$ new cases annually in both developed and developing countries (1-3). It is characterized by the development of a specific pattern of visual field loss and optic neuropathy. Patients are usually treated using filtration surgery which serves to reduce intraocular pressure in the eye (4). Other treatments include topical medication, laser treatment and surgical modalities. Of the treatments available, surgery is the most effective $(5,6)$. Glaucoma filtration surgery may stimulate conjunctival fibroblast proliferation, migration, differentiation and promote ECM secretion, which are important processes in wound healing and scar formation (7-9). However, glaucoma filtration surgery is not always effective, and excessive scarring of the filtering bleb after surgery is a major problem following surgery.

Scar formation at the filtering bleb after glaucoma filtration surgery is a multifactorial process; human conjunctival fibroblasts (HConFs) play an essential role in subconjunctival wound healing (10). In addition, the cytokine transforming growth factor (TGF) $-\beta$ is an important factor in regulating wound healing and scar formation (10). The binding of TGF- $\beta$ to its heterodimeric receptor activates intracellular signaling cascades, including the canonical SMAD pathway and the mitogen-activated protein kinase (MAPK) pathway, and leads to fibrosis (11). The PI3K/AKT signaling pathway, which regulates survival, was also reported to function in regulating cell migration, proliferation and apoptosis $(12,13)$. The PI3K/AKT pathway can be activated by TGF- $\beta$ and also plays an important role in modulating ECM synthesis (14).

All-trans-retinoic acid (ATRA), a derivative of vitamin A, can inhibit TGF- $\beta$ and inhibit fibrosis (15). In a previous study, it was reported that ATRA inhibited TGF- $\beta$-induced HConF-mediated collagen gel contraction via the SMAD signaling pathway (16). However, the effects of ATRA on 
TGF- $\beta$-induced HConF migration, proliferation and ECM synthesis remain unclear. In the present study, HConFs were cultured to investigate the effects of ATRA on the proliferation, migration, apoptosis and ECM synthesis in conjunctival fibroblasts. The aim of the present study was to improve understanding of the mechanism of action of ATRA. The mechanistic insights provided by this study may be applicable to preventing scar formation following glaucoma filtration surgery.

\section{Materials and methods}

Materials. ATRA and TGF- $\beta$ (Sigma-Aldrich; Merck KGaA) were prepared as 0.4 and $50 \mathrm{ng} / \mathrm{ml}$ stock solutions in DMSO, respectively, and were stored below $-20^{\circ} \mathrm{C}$ in the dark. ATRA and TGF- $\beta$ were diluted to $10-200 \mu \mathrm{M}$ and $1 \mathrm{ng} / \mathrm{ml}$, respectively, in DMEM (Sigma-Aldrich; Merck KGaA) containing 10\% FBS (Gibco; Thermo Fisher Scientific, Inc.) on the day of the experiment. The highest concentration of DMSO in the test solutions was $0.1 \%$. To exclude the possibility that proliferation may be inhibited by DMSO, all cells were exposed to a final DMSO concentration of $0.1 \%$ in DMEM containing 10\% FBS.

Cell culture. Primary HConFs (ScienCell Research Laboratories, Inc.) were cultured according to the manufacturer's instructions; three individual lots of HConFs were obtained. The $\mathrm{HConFs}$ were maintained and cultured at $37^{\circ} \mathrm{C}$ in fibroblast medium (ScienCell Research Laboratories, Inc.) containing 2\% FBS, fibroblast growth supplement (ScienCell Research Laboratories, Inc.), $100 \mathrm{U} / \mathrm{ml}$ penicillin and $100 \mu \mathrm{g} / \mathrm{ml}$ streptomycin in a $5 \% \mathrm{CO}_{2}$ humidified incubator. The $\mathrm{HConFs}$ were characterized by a spindle-shaped morphology and were positively stained with anti-fibronectin antibodies.

Cell proliferation assay. A HConF proliferation assay was performed as previously described (17). Briefly, HConF suspensions $\left(0.5 \times 10^{4}\right.$ cells/well $)$ were cultured in fibroblast medium in a 96 -well plate $(200 \mu \mathrm{l} /$ well $)$ for $24 \mathrm{~h}$ at $37^{\circ} \mathrm{C}$. ATRA $(0.01-10 \mu \mathrm{M})$ was added to the fibroblast medium and the cells were cultured for a further $48 \mathrm{~h}$ at $37^{\circ} \mathrm{C}$ in the absence or presence of TGF- $\beta(1 \mathrm{ng} / \mathrm{ml})$, with $0.1 \%$ DMSO as the control. The concentrations of ATRA and TGF- $\beta$ used were determined according to a previous study (15). Subsequently, each well was incubated with $10 \mu \mathrm{l}$ Cell Counting Kit- 8 solution (BestBio; Sigma) for a further $3 \mathrm{~h}$ at $37^{\circ} \mathrm{C}$. The absorbance was measured at $450 \mathrm{~nm}$ using an automated microplate reader (model 3001-1387; Thermo Fisher Scientific, Inc.).

Apoptosis assay. HConF apoptosis was determined using an Annexin V-FITC apoptosis kit (BestBio) as previously described (17). Briefly, the HConFs $\left(5 \times 10^{5}\right.$ cells/well) were plated in 6-well plates and cultured for $48 \mathrm{~h}$. After rinsing twice with PBS, the cells were resuspended in $400 \mu 1 \mathrm{1X}$ binding buffer [10 mM HEPES, $140 \mathrm{mM}$ sodium chloride, $2.5 \mathrm{mM}$ calcium chloride ( $\mathrm{pH}$ 7.4)], into which $5 \mu 1$ of Annexin V-FITC was added. After 15 min incubation at $2-8^{\circ} \mathrm{C}$ in the dark, $10 \mu \mathrm{l}$ of propidium iodide (BestBio) was added to the cells prior to further incubation for $5 \mathrm{~min}$ at $2-8^{\circ} \mathrm{C}$ in the dark. Within $15 \mathrm{~min}$ after staining, the cells were analyzed using a flow cytometer (Cytomics FV 500) and CytExpert 2.0 software (both Beckman Coulter, Inc.). The apoptotic rate was calculated as the sum of the percentage of early + late apoptotic cells.

Wound healing assay. HConFs were seeded at a density of $5 \times 10^{5}$ cells/well in 6 -well plates. The culture medium for the HConFs was replaced after $24 \mathrm{~h}$ with serum-free medium, and then cells were incubated for a further $2 \mathrm{~h}$ at $37^{\circ} \mathrm{C}$. Next, a sterile $20-\mu \mathrm{l}$ pipette tip was used to scratch a line in the confluent cell monolayer, after which the cells were washed three times with PBS. The scratch wound healed after $24 \mathrm{~h}$ of incubation in the following conditions: $1 \mu \mathrm{M}$ ATRA $+1 \mathrm{ng} / \mathrm{ml}$ TGF- $\beta, 1 \mathrm{ng} / \mathrm{ml}$ TGF- $\beta$ and fibroblast medium without ATRA and TGF- $\beta$. The effects on HConF migration were evaluated by measuring the area of the wound from the images captured at a magnification of $\mathrm{x} 100$ at 0 and $24 \mathrm{~h}$ using a light microscope (Olympus Corporation); analysis was performed using ImageJ version 1.5 (National Institutes of Health).

Transwell migration assay. A chemomigration assay was performed using Transwell plates (pore size, $8 \mu \mathrm{m}$ ) as previously described (18). Briefly, the cells in the upper Transwell chamber $\left(1 \times 10^{5}\right)$ were suspended in $200 \mu \mathrm{l}$ of DMEM, and DMEM containing 20\% FBS was added to the lower chamber. Following a 12 -h incubation at $37^{\circ} \mathrm{C}$, the medium with non-migrated cells in the upper chamber was removed with a cotton swab. The cells that had migrated to the lower chamber were fixed with $4 \%$ paraformaldehyde for $30 \mathrm{~min}$ at $37^{\circ} \mathrm{C}$ and then stained with $0.5 \%$ crystal violet for $10 \mathrm{~min}$ at $37^{\circ} \mathrm{C}$. The cells were counted at $\times 200$ magnification using a light microscope in six different fields of view.

Cell treatment. HConFs were incubated in fibroblast medium in 6-well plates for $24 \mathrm{~h}$. Then, the medium was replaced with the serum-free medium. Serum-starved cells were incubated for $6 \mathrm{~h}$ in the presence or absence of ATRA $(1 \mu \mathrm{M})$, and then for $48 \mathrm{~h}$ in the presence or absence of TGF- $\beta(1 \mathrm{ng} / \mathrm{ml})$. Then, cell lysates were analyzed via western blotting.

Western blot analysis. Western blot analysis was performed as previously described $(17,19,20)$. Cells were washed with pre-cooled PBS and lysed with RIPA buffer (Nanjing KeyGen Biotech Co., Ltd.) containing the protease inhibitor phenylmethylsulfonyl fluoride and the phosphatase inhibitor sodium orthovanadate (Nanjing KeyGen Biotech Co., Ltd.) on ice for $30 \mathrm{~min}$. Subsequently, the cells were gently scraped from the plate and centrifuged at $1,500 \mathrm{xg}$ for $12 \mathrm{~min}$ at $4^{\circ} \mathrm{C}$. Protein concentrations were measured using the bicinchoninic acid method. A total of $20 \mu \mathrm{g} / \mathrm{sample}$ of protein was separated via 6-15\% SDS-PAGE and then transferred onto PVDF membranes. Membranes were blocked with 5\% non-fat dry milk for $1 \mathrm{~h}$ at $37^{\circ} \mathrm{C}$ before overnight incubation at $4^{\circ} \mathrm{C}$ with mouse monoclonal anti-collagen I (1:2,500; cat. no. ab88147; Abcam), rabbit polyclonal anti-fibronectin (1:1,500; cat. no. ab137720; Abcam), mouse antibodies against PI3K p85 $\alpha$ (1:500; cat. no. sc-1637), phosphorylated (p)-PI3K p85 $\alpha$ (1:500; cat. no. sc-12929), AKT (1:500; cat. no. sc-5298) and p-AKT (1:500; cat. no. sc-293125; all from Santa Cruz Biotechnology, Inc.), and mouse monoclonal anti-GAPDH (1:1,000; cat. no. A01020; Abbkine Scientific Co., Ltd.). Each membrane was incubated with 
anti-rabbit (1:5,000; cat. no. A0208) or anti-mouse (1:5,000; cat. no. A0216) horseradish peroxidase-conjugated secondary antibodies (both from Beyotime Institute of Biotechnology) for $1 \mathrm{~h}$ at room temperature. The blots were visualized using ECL reagent (Beyotime Institute of Biotechnology) and the bands were analyzed using ImageJ software.

Inhibition of TGF- $\beta$-induced collagen I and fibronectin expression in HConFs using a PI3K inhibitor. HConFs were incubated in fibroblast medium in 6-well plates for $24 \mathrm{~h}$, then the medium was replaced with serum-free medium. Serum-starved cells were incubated with $10 \mu \mathrm{M}$ LY294002 (Calbiochem; Merck KGaA) for $1 \mathrm{~h}$ at $37^{\circ} \mathrm{C}$. Subsequently, the cells were treated with $1 \mathrm{ng} / \mathrm{ml}$ TGF- $\beta$ for $48 \mathrm{~h}$ at $37^{\circ} \mathrm{C}$. The effects of the PI3K inhibitor on the TGF- $\beta$-induced expression of collagen I and fibronectin in HConF were investigated via western blot analysis.

Statistical analysis. Data analysis was performed using SPSS version 19.0 (IBM Corp.). The data are presented as the mean \pm SD. Each experiment was repeated at least three times. Statistical analysis was performed using Student's t-test or one-way ANOVA; following ANOVA, the least significant difference test was used for pairwise comparisons between groups. $\mathrm{P}<0.05$ was considered to indicate a statistically significant difference.

\section{Results}

ATRA inhibits TGF- $\beta$-induced HConF proliferation. The effect of ATRA on HConF proliferation was examined. The cells were incubated for $48 \mathrm{~h}$ with $0.01-10 \mu \mathrm{M}$ ATRA in the absence or presence of $1 \mathrm{ng} / \mathrm{ml}$ TGF- $\beta$ with $0.1 \%$ DMSO as the control. ATRA was revealed to inhibit HConF proliferation in a concentration-dependent manner $(\mathrm{P}<0.05$; Fig. 1A). HConFs were divided into three treatment groups: The ATRA group $(1 \mu \mathrm{M}$ ATRA $+1 \mathrm{ng} / \mathrm{ml}$ TGF- $\beta)$, the TGF- $\beta$ group ( $1 \mathrm{ng} / \mathrm{ml}$ TGF- $\beta$ ) and the control group (0.1\% DMSO). ATRA inhibited TGF- $\beta$-induced HConF proliferation by $77.50 \pm 1.88 \%$ ( $\mathrm{P}<0.01$ vs. TGF- $\beta$; Fig. $1 \mathrm{~B})$.

ATRA induces HConF apoptosis. The effect of ATRA on HConF apoptosis was examined using flow cytometry. A significantly increased number of apoptotic HConFs were observed in the ATRA group compared with the control group (53.25 \pm 1.2 vs. $22.5 \pm 1.1 \%$, respectively; $\mathrm{P}<0.01)$. The TGF- $\beta$ group showed a significantly decreased apoptotic rate compared with the control group $(14.75 \pm 1.4 \%$; $\mathrm{P}<0.01$; Fig. 2$)$.

ATRA inhibits TGF- $\beta$-induced cell migration. The migratory ability of the HConFs was evaluated using in vitro wound healing and Transwell assays. Similar results were observed for the two assays. The ATRA group exhibited a $\sim 30 \%$ reduction in wound healing compared with the control group $(48.9 \pm 1.34$ vs. $71.30 \pm 1.55 \%$, respectively; $\mathrm{P}<0.05)$. Wound healing was increased in the TGF- $\beta$ group compared with the control group $(90.50 \pm 1.22 \%$; $\mathrm{P}<0.05 ;$ Fig. 3A). Reduced HConF migration was observed for the ATRA group (14.85 \pm 1.13 cells; $\mathrm{P}<0.01)$, whereas significantly increased migration was noted for the TGF- $\beta$ group compared with the control group
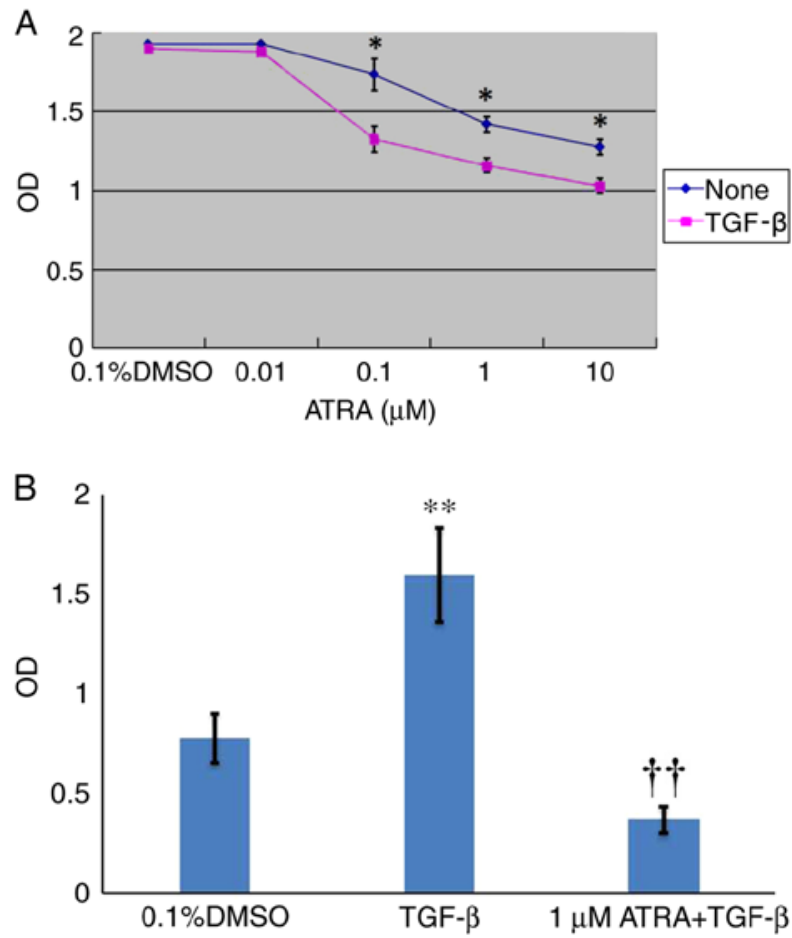

Figure 1. Effect of ATRA on HConF proliferation. Cells were cultured for $48 \mathrm{~h}$ in the presence or absence of TGF- $\beta(1 \mathrm{ng} / \mathrm{ml})$ and in the presence of the indicated concentrations of ATRA. The relative cell numbers were detected with a Cell Counting Kit-8 assay. (A) Concentration dependency of ATRA on TGF- $\beta$-induced HConFs proliferation ${ }^{*} \mathrm{P}<0.05$ vs. TGF- $\beta$. (B) HConFs were divided into 3 groups: ATRA group (treated with $1 \mu \mathrm{M}$ ATRA + $1 \mathrm{ng} / \mathrm{ml}$ TGF- $\beta$ ), TGF- $\beta$ group (treated with $1 \mathrm{ng} / \mathrm{ml}$ TGF- $\beta$ ), and $0.1 \%$ DMSO control group. Data are presented as the mean \pm SD. ${ }^{* *} \mathrm{P}<0.01$ vs. $0.1 \%$ DMSO; ${ }^{\dagger} \mathrm{P}<0.01$ vs TGF- $\beta$. ATRA, all-trans-retinoic acid; HConFs, human conjunctival fibroblasts; TGF- $\beta$, transforming growth factor- $\beta$.

(135.55 \pm 1.12 cells vs. $28.65 \pm 1.02$ cells, respectively; $\mathrm{P}<0.01$; Fig. 3B) in the Transwell migration assays.

ATRA inhibits TGF- $\beta$-induced collagen I and fibronectin expression. The effect of ATRA on TGF- $\beta$-induced ECM production was investigated. Western blotting analysis and densitometric analysis revealed that exposure of HConFs to $1 \mathrm{ng} / \mathrm{ml} \mathrm{TGF}-\beta$ for $48 \mathrm{~h}$ induced the production of collagen I and fibronectin, whereas $1 \mu \mathrm{M}$ ATRA inhibited TGF- $\beta$-induced synthesis of collagen I and fibronectin by HConFs ( $\mathrm{P}<0.01$; Fig. 4).

ATRA inhibits the PI3K/AKT pathway. The effect of ATRA on TGF- $\beta$-induced PI3K and AKT phosphorylation in HConFs was examined (Fig. 5). Western blotting analysis and densitometric analysis revealed that the exposure of HConFs to $1 \mathrm{ng} / \mathrm{ml}$ TGF- $\beta$ for $48 \mathrm{~h}$ induced the phosphorylation/activation of PI3K and AKT. Treatment with $1 \mu \mathrm{M}$ ATRA inhibited TGF- $\beta$-induced PI $3 \mathrm{~K}$ and AKT phosphorylation, indicating that ATRA inhibited the TGF- $\beta$-induced PI3K/AKT signaling pathway $(\mathrm{P}<0.05$; Fig. 5).

Inhibition of TGF- $\beta$-induced collagen I and fibronectin expression in HConFs by a PI3K inhibitor. The effect of the PI3K inhibitor LY294002 on the TGF- $\beta$-induced expression of collagen I and fibronectin in HConF was investigated. Cells were incubated with $10 \mu \mathrm{M} \mathrm{LY} 294002$ for $1 \mathrm{~h}$ before treatment 

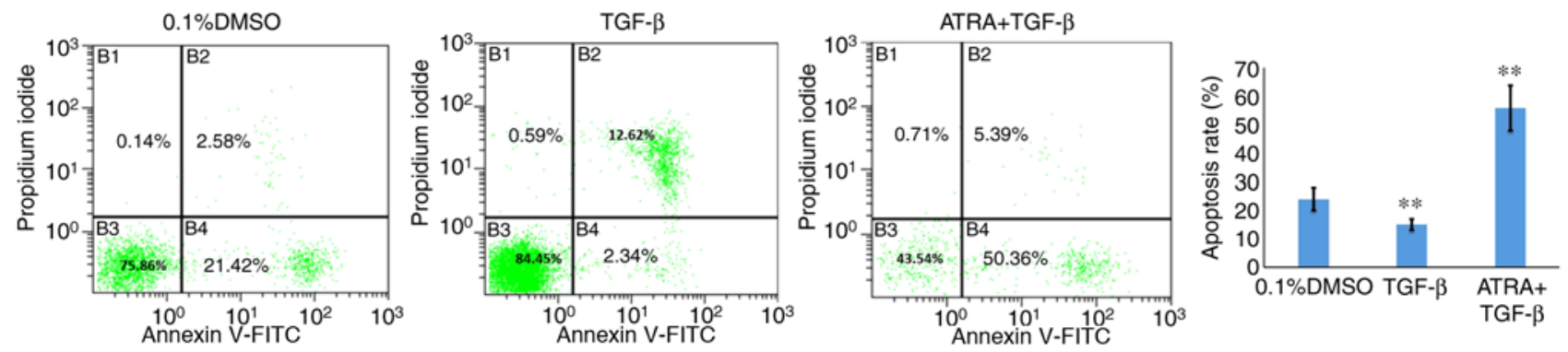

Figure 2. Effect of ATRA on HConF apoptosis. HConFs were cultured with $1 \mathrm{ng} / \mathrm{ml}$ TGF- $\beta$ or $1 \mu \mathrm{M}$ ATRA $+1 \mathrm{ng} / \mathrm{ml}$ TGF- $\beta$ for 48 h. Cells were stained with propidium iodide (y-axis) and Annexin V-FITC (x-axis), and analyzed by flow cytometry. For each plot, the upper right quadrant represents late apoptotic cells and the lower right quadrant represents early apoptotic cells. The total apoptosis rate was calculated by the addition of the early apoptosis and the late apoptosis values. Data are presented as the mean \pm SD. ${ }^{* *} \mathrm{P}<0.01$; vs. $0.01 \%$ DMSO. ATRA, all-trans-retinoic acid; HConFs, human conjunctival fibroblasts; TGF- $\beta$, transforming growth factor- $\beta$.

A
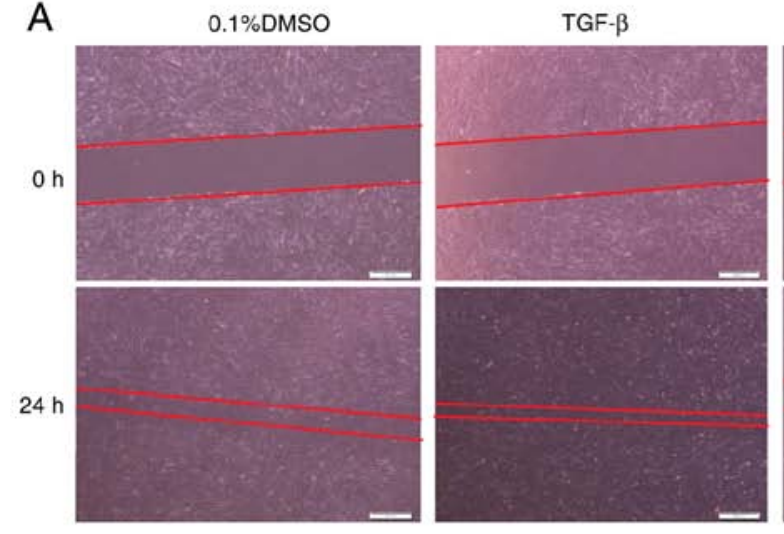

B
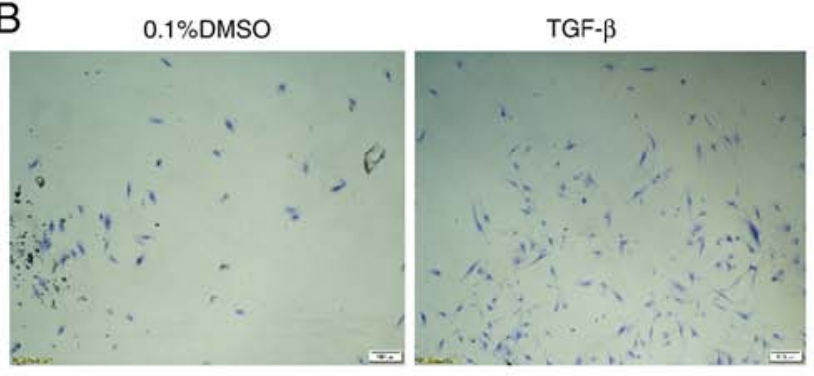

ATRA+TGF- $\beta$
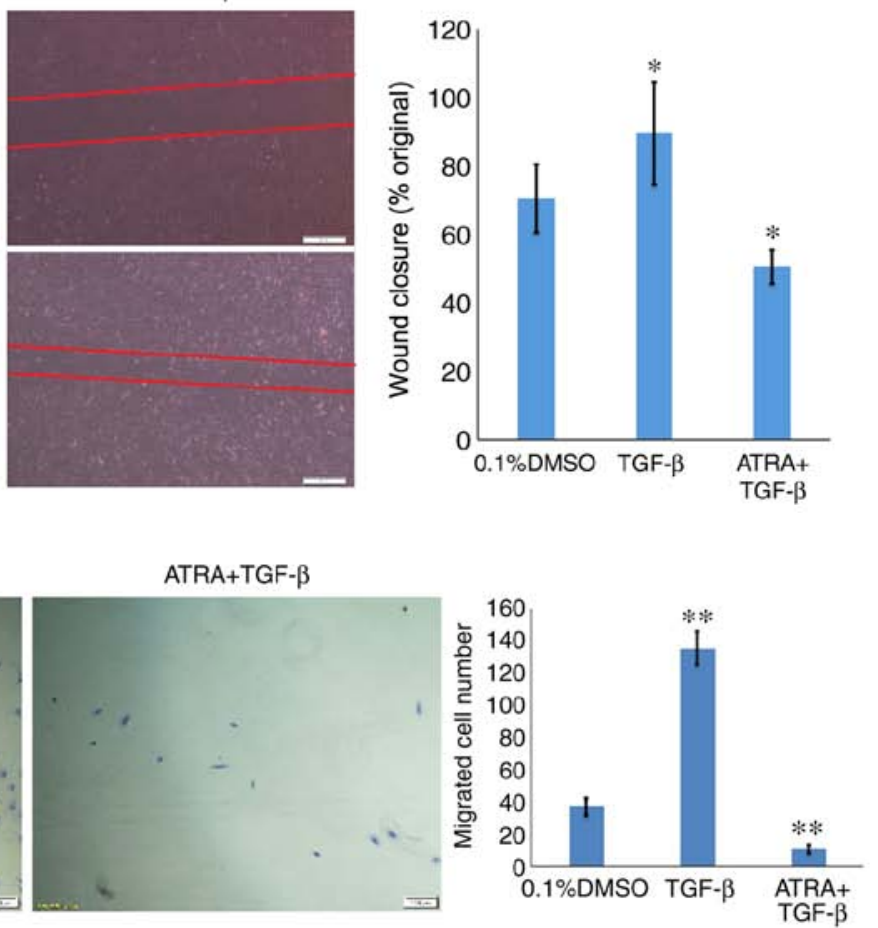

Figure 3. Effect of ATRA on TGF- $\beta$-induced HConF migration. The effect of ATRA on HConF migration was assessed using in vitro wound healing and Transwell assays. The migratory ability of the HConFs was determined by measuring the width of the wound after $24 \mathrm{~h}$ or the number of cells that had migrated to the lower chamber after $12 \mathrm{~h}$. (A) Representative images of the wound healing assay results and quantification of the inhibitory effect of ATRA on HConF migration; magnification: x100. (B) Representative images of the Transwell migration assay and quantification of the inhibitory effect of ATRA on HConF migration; magnification: $\mathrm{x} 200$. Data are presented as the mean $\pm \mathrm{SD} .{ }^{*} \mathrm{P}<0.05,{ }^{* *} \mathrm{P}<0.01$ vs. $0.01 \%$ DMSO. ATRA, all-trans-retinoic acid; HConFs, human conjunctival fibroblasts; TGF- $\beta$, transforming growth factor- $\beta$.

with $1 \mathrm{ng} / \mathrm{ml}$ TGF- $\beta$ for $48 \mathrm{~h}$. Western blotting analysis and densitometric analysis revealed that LY294002 significantly inhibited TGF- $\beta$-induced expression of collagen I and fibronectin in HConFs $(\mathrm{P}<0.05$; Fig. 6$)$. The TGF- $\beta$-induced expression of collagen I and fibronectin in HConFs was inhibited by 27 and 47\% in the presence of LY294002, respectively. The inhibitor had no effect on collagen I and fibronectin expression in the absence of TGF- $\beta$ (data not shown).

\section{Discussion}

In the present study, it was revealed that ATRA inhibited TGF- $\beta$-induced migration, proliferation and ECM synthesis in
HConFs. Furthermore, ATRA was found to promote apoptosis and inhibit the TGF- $\beta$-induced phosphorylation of PI3K and AKT in HConFs. In addition, the present study showed that the PI3K inhibitor LY294002 attenuated the TGF- $\beta$-induced expression of collagen I and fibronectin.

Preventing conjunctival scarring remains a challenge in clinical ophthalmology. TGF- $\beta$ is a key factor implicated in postoperative scarring as it stimulates the migration, proliferation and differentiation HConFs, and promotes ECM deposition and remodeling (21). ATRA, a derivative of vitamin A, has been shown to regulate ECM expression and serve an important role in fibrotic diseases through the inhibition of TGF- $\beta 1$ (22). Moreover, ATRA was found to 


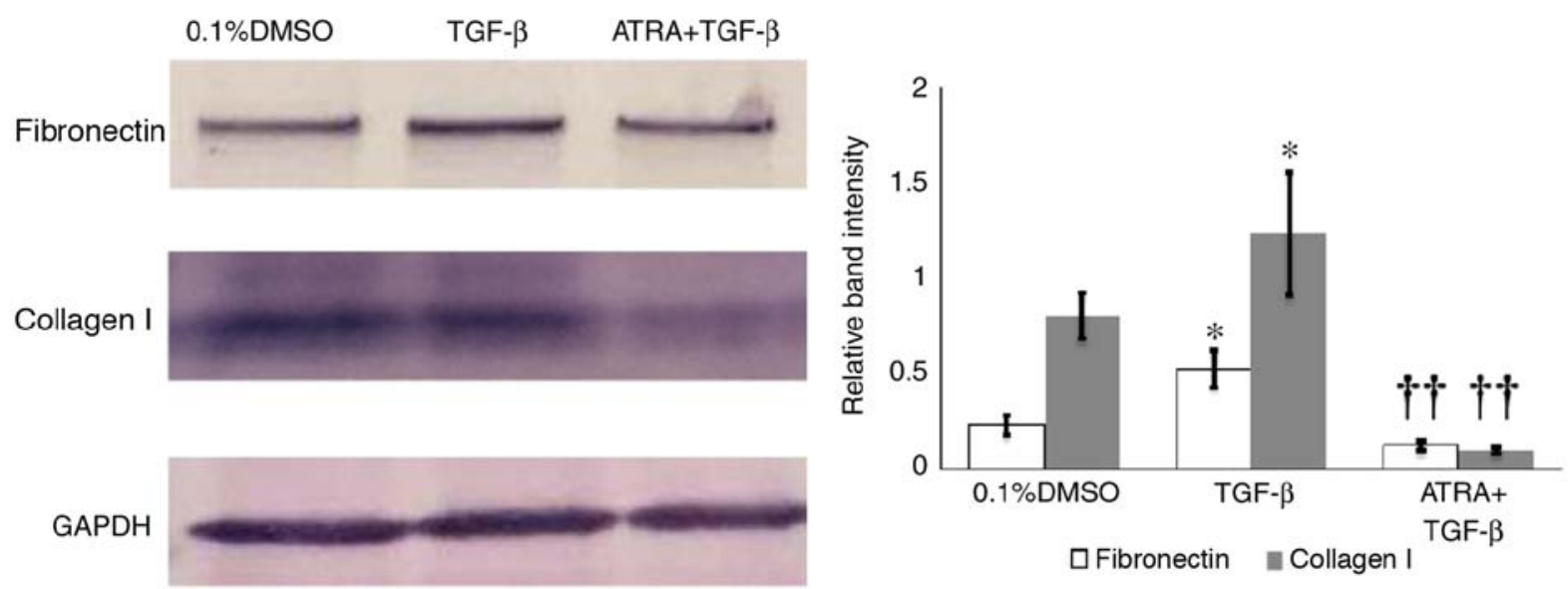

Figure 4. Effect of ATRA on TGF- $\beta$-induced collagen I and fibronectin expression in HConFs. Serum-deprived cells were incubated for $6 \mathrm{~h}$ in the absence or presence of ATRA $(1 \mu \mathrm{M})$ and then for $48 \mathrm{~h}$ in the absence or presence of TGF- $\beta(1 \mathrm{ng} / \mathrm{ml})$. Cell lysates were subjected to western blot analysis with collagen I, fibronectin and GAPDH antibodies. The data are representative of three independent experiments. The western blots were subjected to densitometric analysis in order to determine the intensity of the bands corresponding to collagen I and fibronectin. The data are expressed relative to the intensity of the corresponding GAPDH band. Data are presented as the mean \pm SD. ${ }^{*} \mathrm{P}<0.05$ vs. $0.1 \%$ DMSO; ${ }^{\dagger} \mathrm{P}<0.01$ vs. TGF- $\beta$. ATRA, all-trans-retinoic acid; HConFs, human conjunctival fibroblasts; TGF- $\beta$, transforming growth factor- $\beta$.
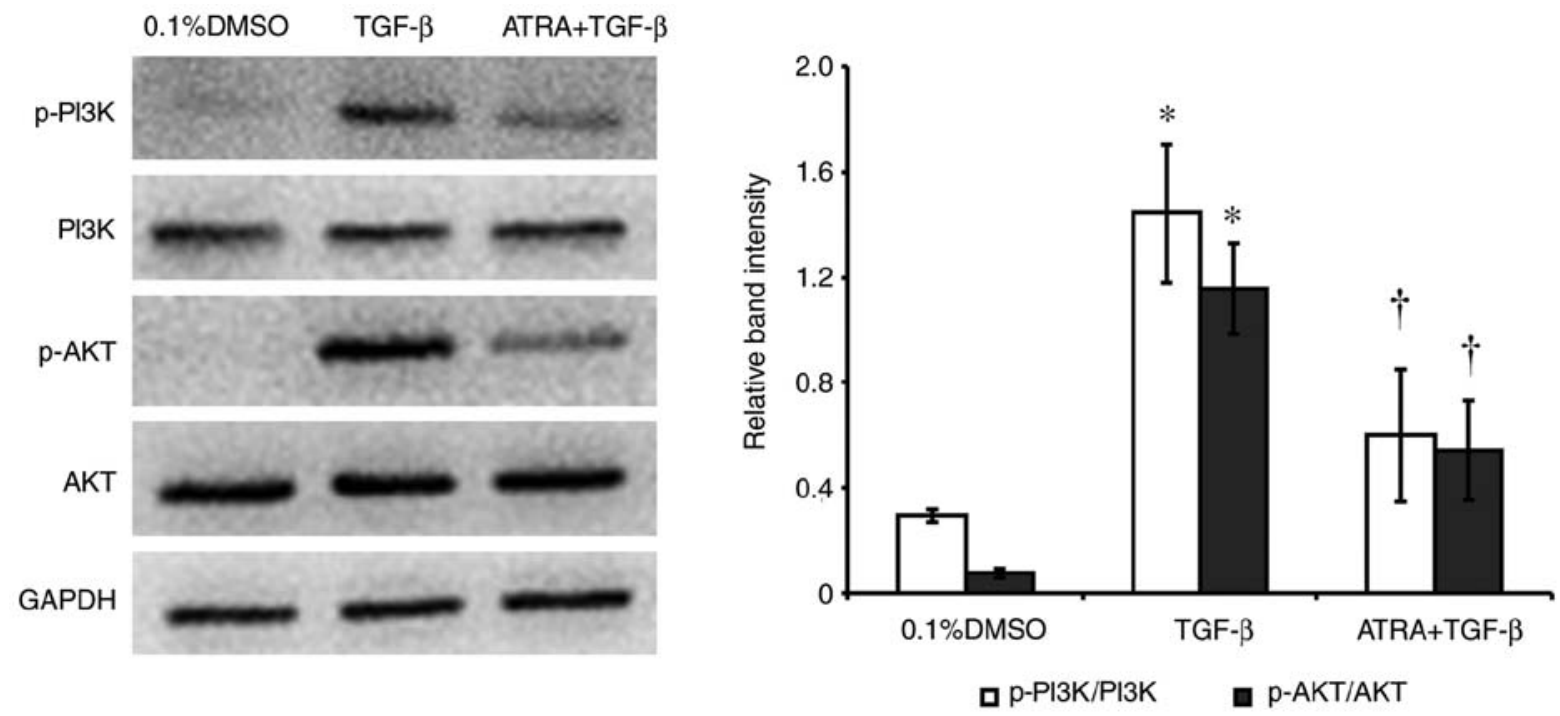

Figure 5. ATRA-mediated inhibition of TGF- $\beta$-induced p-PI3K and p-AKT in HConFs. Serum-deprived cells were incubated for $6 \mathrm{~h}$ in the presence or absence of ATRA $(1 \mu \mathrm{M})$ and then for $48 \mathrm{~h}$ in the presence or absence of TGF- $\beta(1 \mathrm{ng} / \mathrm{ml})$. Cell lysates were subjected to western blot analysis with p-PI3K, PI3K, p-AKT, AKT and GAPDH antibodies. The data are representative of three independent experiments. The western blots were subjected to densitometric analysis in order to determine the intensity of the bands corresponding to p-PI3K and p-AKT. The data are expressed relative to the intensity of the corresponding GAPDH band. Data are presented as the mean $\pm \mathrm{SD}$. " $\mathrm{P}<0.05$ vs. $0.1 \%$ DMSO; ${ }^{\dagger} \mathrm{P}<0.05$ vs. TGF- $\beta$. ATRA, all-trans-retinoic acid; HConFs, human conjunctival fibroblasts; TGF- $\beta$, transforming growth factor- $\beta ; p$, phosphorylated.

serve a protective role against collagen accumulation, cell injury and proliferation in various types of fibrosis, including liver fibrosis, pulmonary fibrosis and kidney fibrosis (22-25). In a previous study, it was demonstrated that ATRA inhibited TGF- $\beta$-induced HConF-mediated collagen gel contraction by attenuating the formation of actin stress fibers and focal adhesions, as well as by inhibiting MAPK, c-Jun and SMAD signaling (16). In the present study, it was demonstrated that ATRA inhibited the TGF- $\beta$-induced migration and proliferation of HConFs, inhibited ECM synthesis and increased the apoptosis in HConFs. Collectively, these results suggested that ATRA attenuates conjunctival scarring by modulating the function of HConFs and ECM synthesis.
The PI3K/AKT pathway modulates cell proliferation, differentiation, apoptosis, motility, survival and glucose metabolism (26). PI3K generates 3'-phosphorylated phosphoinositides, including phosphatidylinositol-3,4-bisphosphate and phosphatidylinositol-3,4,5-triphosphate, which then recruit target proteins to the plasma membrane (27). AKT is a serine/threonine kinase that acts as an effector of PI3K (27). It has been reported that activation of the PI3K/AKT signaling pathway may induce ECM secretion in several cell types $(28,29)$. LY294002 and AKT small interfering RNA were reported to significantly reduce TGF- $\beta 1$-induced $\alpha$-smooth muscle actin expression, a marker of fibroblasts, in conjunctival fibroblasts, indicating that these changes were mediated by the PI3K/AKT 

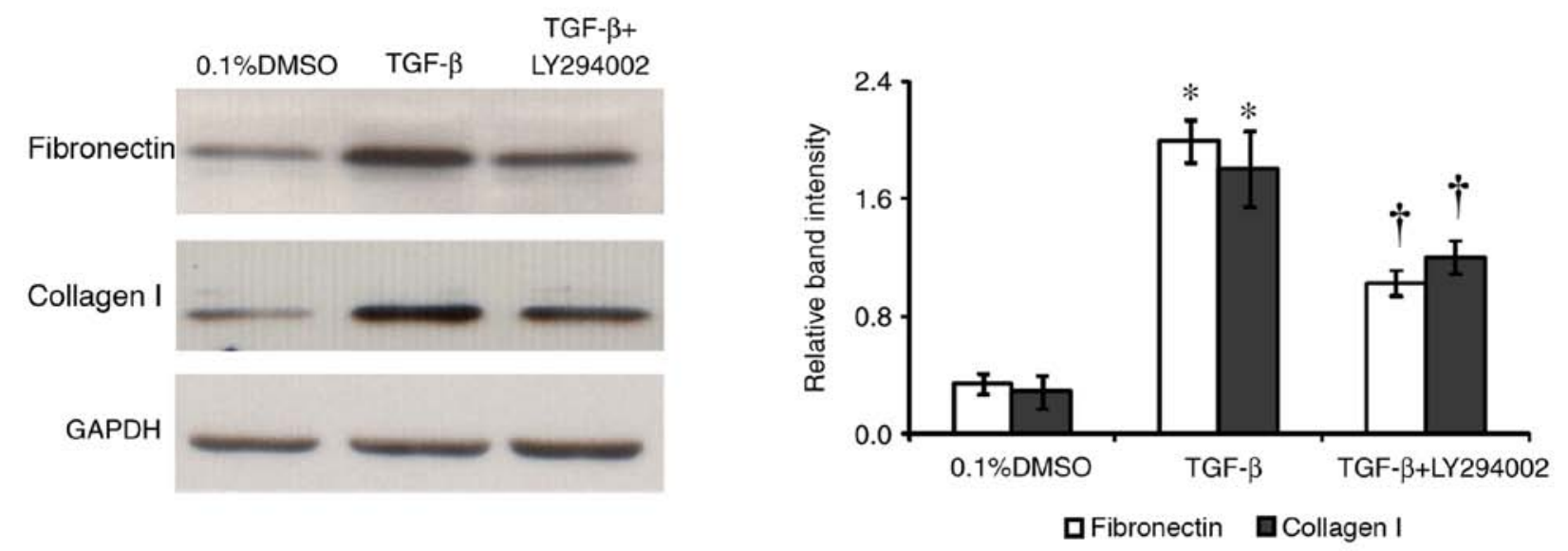

Figure 6. Effect of PI3K inhibition on TGF- $\beta$-induced expression of collagen I and fibronectin in HConFs. Serum-deprived cells were incubated for $1 \mathrm{~h}$ in the presence or absence of the PI3K inhibitor LY294002 $(10 \mu \mathrm{M})$ and then for $48 \mathrm{~h}$ in the presence or absence of TGF- $\beta(1 \mathrm{ng} / \mathrm{ml})$. Cell lysates were subjected to western blot analysis with collagen I, fibronectin or and GAPDH antibodies. The data are representative of three independent experiments. The western blots were subjected to densitometric analysis to determine the intensity of the bands corresponding to collagen I and fibronectin. The data are expressed relative to the intensity of the corresponding GAPDH band. Data are presented as the mean $\pm \mathrm{SD}$. " $\mathrm{P}<0.05$ vs. $0.1 \%$ DMSO; ${ }^{\dagger} \mathrm{P}<0.05$ vs. TGF- $\beta$. ATRA, all-trans-retinoic acid; HConFs, human conjunctival fibroblasts; TGF- $\beta$, transforming growth factor- $\beta$.

pathway (30). In addition, LY294002 could inhibit the proliferation and migration of conjunctival fibroblasts $(31,32)$. In the present study, it was shown that ATRA promoted apoptosis and inhibited proliferation, migration and ECM synthesis. In addition, it was demonstrated that LY294002 could inhibit TGF- $\beta$-induced expression of collagen I and fibronectin, similar to ATRA; this is consistent with the results of a previous study (33). Hence, it was deduced that the inhibitory effects of ATRA on HConFs are likely mediated by inhibition of the PI3K/AKT signaling pathway.

TGF- $\beta$-mediated signaling can occur via SMAD-dependent or SMAD-independent pathways; the SMAD-independent pathway includes the MAPK and PI3K/AKT pathways (34). TGF- $\beta$ activates the SMAD pathway via the phosphorylation of Smad2 and Smad3, which then leads to the formation of a Smad2/3 complex; the Smad2/3 complex and Smad4 are subsequently translocated to the nucleus in order to regulate the expression of genes associated with fibroblast proliferation, migration and ECM deposition (35). In our previous study, a role for SMAD signaling was found in the ATRA-mediated inhibition of TGF- $\beta$-induced, HConF-mediated collagen gel contraction (16). Therefore, it was hypothesized that the SMAD signaling pathway is also involved in the inhibitory effects of ATRA on proliferation, migration and ECM synthesis in HConFs.

In conclusion, the findings of the present study revealed the mechanism of action of ATRA. ATRA was found to modulate PI3K/AKT signaling and impact on TGF- $\beta$-induced apoptosis, proliferation, migration and ECM synthesis. Given the significant global impact of glaucoma and the inadequacy in its treatment methods, conjunctival scarring at the surgical site after glaucoma filtration surgery $(36,37)$ and the adverse side effects of anti-scarring anti-metabolite medication, such as mitomycin-C and 5-fluorouracil, which can potentially lead to blindness (38-41), there is a requirement for improved therapeutic approaches. From the present study, it is proposed that ATRA may be a promising compound capable of modulating scar formation after glaucoma filtration surgery. However, as the present study was performed in vitro, future research should be directed towards characterizing the in vivo effects of ATRA.

\section{Acknowledgements}

Not applicable.

\section{Funding}

The present study was funded by the National Natural Science Foundation of China (grant no. 81770889), the Natural Science Foundation of Guangdong Province (grant no. 2017A030313774) and the Health Programs of Finance Department of Jilin Province (grant no. 3D5177783429).

\section{Availability of data and materials}

All the data generated and analyzed in the present study are available from the corresponding author upon reasonable request.

\section{Authors' contributions}

$Y L$ and $Y Z$ conceived and designed the experiments. LL and $\mathrm{XW}$ performed the experiments. LL, XW, and YZ analyzed the data. LL and YL wrote the manuscript. All the authors have read and approved the final version of the manuscript.

\section{Ethics approval and consent to participate}

Not applicable.

\section{Patient consent for publication}

Not applicable.

\section{Competing interests}

The authors declare that they have no competing interests. 


\section{References}

1. Thylefors B and Négrel AD: The global impact of glaucoma Bull World Health Org 72: 323-326, 1994.

2. Foster A and Johnson GJ: Magnitude and causes of blindness in the developing world. Int Ophthalmol 14: 135-140, 1990.

3. Quigley HA: Number of people with glaucoma worldwide. $\mathrm{Br}$ J Ophthalmol 80: 389-393, 1996.

4. Sommer A: Doyne lecture. Glaucoma: Facts and figures. Eye (Lond) 10: 295-301, 1996.

5. Jay JL: Rational choice of therapy in primary open angle glaucoma. Eye (Lond) 6: 243-247, 1992.

6. Migdal C, Gregory W and Hitchings R: Long-term functional outcome after early surgery compared with laser and medicine in open-angle glaucoma. Ophthalmology 101: 1651-1657, 1994.

7. Chang L, Crowston JG, Cordeiro MF, Akbar AN and Khaw PT: The role of the immune system in conjunctival wound healing after glaucoma surgery. Surv Ophthalmol 45: 49-68, 2000.

8. Atreides SP, Skuta GL and Reynolds AC: Wound healing modulation in glaucoma filtering surgery. Int Ophthalmol Clin 44: 61-106, 2004

9. Desjardins DC, Parrish RK II, Folberg R, Nevarez J, Heuer DK and Gressel MG: Wound healing after filtering surgery in ow monkeys. Arch Ophthalmol 104: 1835-1839, 1986.

10. Saika S, Yamanaka O, Okada Y, Tanaka S, Miyamoto T, Sumioka T, Kitano A, Shirai K and Ikeda K: TGF beta in fibroproliferative diseases in the eye. Front Biosci (Schol Ed) 1: 376-390, 2009

11. Cong M, Iwaisako K, Jiang C and Kisseleva T: Cell Signals Influencing Hepatic Fibrosis. Int J Hepatol 2012: 158547, 2012.

12. Sang $\mathrm{H}$, Li T, Li H and Liu J: Gabl regulates proliferation and migration through the PI3K/Akt signaling pathway in intrahepatic cholangiocarcinoma. Tumour Biol 36: 8367-8377, 2015

13. Zhou H, Li D, Shi C, Xin T, Yang J, Zhou Y, Hu S, Tian F, Wang J and Chen Y: Effects of exendin-4 on bone marrow mesenchymal stem cell proliferation, migration and apoptosis in vitro. Sci Rep 5: 12898, 2015.

14. Zeng R, Xiong Y, Zhu F, Ma Z, Liao W, He Y, He J, Li W Yang J, Lu Q, et al: Fenofibrate attenuated glucose-induced mesangial cells proliferation and extracellular matrix synthesis via PI3K/AKT and ERK1/2. PLoS One 8: e76836, 2013.

15. Wen X, Li Y, Hu K, Dai C and Liu Y: Hepatocyte growth factor receptor signaling mediates the anti-fibrotic action of 9-cisretinoic acid in glomerular mesangial cells. Am J Pathol 167: 947-957, 2005 .

16. Liu Y, Kimura K, Orita T, Teranishi S, Suzuki K and Sonoda KH Inhibition by all-trans-retinoic acid of transforming growth factor- $\beta$-induced collagen gel contraction mediated by human tenon fibroblasts. Invest Ophthalmol Vis Sci 55: 4199-4205, 2014.

17. Sun L, Dong Y, Zhao J, Yin Y, Tong B, Zheng Y and Xin $\mathrm{H}$ NPPB modulates apoptosis, proliferation, migration and extracellular matrix synthesis of conjunctival fibroblasts by inhibiting PI3K/AKT signaling. Int J Mol Med 41: 1331-1338, 2018

18. Marshall J: Transwell(®) invasion assays. Methods Mol Biol 769: 97-110, 2011

19. Liu Y, Ko JA, Yanai R, Kimura K, Chikama T, Sagara T and Nishida T: Induction by latanoprost of collagen gel contraction mediated by human tenon fibroblasts: Role of intracellular signaling molecules. Invest Ophthalmol Vis Sci 49: 1429-1436, 2008.

20. Liu Y, Yanai R, Lu Y, Kimura K and Nishida T: Promotion by fibronectin of collagen gel contraction mediated by human corneal fibroblasts. Exp Eye Res 83: 1196-1204, 2006.

21. Cordeiro MF: Role of transforming growth factor beta in conjunctival scarring. Clin Sci (Lond) 104: 181-187, 2003

22. Ye Y and Dan Z: All-trans retinoic acid diminishes collagen production in a hepatic stellate cell line via suppression of active protein-1 and c-Jun N-terminal kinase signal. J Huazhong Univ Sci Technolog Med Sci 30: 726-733, 2010.
23. Dong Z, Tai W, Yang Y, Zhang T, Li Y, Chai Y, Zhong H, Zou $\mathrm{H}$ and Wang D: The role of all-trans retinoic acid in bleomycin-induced pulmonary fibrosis in mice. Exp Lung Res 38: 82-89, 2012.

24. Morath C, Dechow C, Lehrke I, Haxsen V, Waldherr R, Floege J, Ritz E and Wagner J: Effects of retinoids on the TGF-beta system and extracellular matrix in experimental glomerulonephritis. J Am Soc Nephrol 12: 2300-2309, 2001.

25. Zhou TB, Drummen GP and Qin YH: The controversial role of retinoic acid in fibrotic diseases: Analysis of involved signaling pathways. Int J Mol Sci 14: 226-243, 2013.

26. Chen J: The IL-23/IL-17 axis may be important in obesity-asso-ciated cancer by way of the activation of multiple signal pathways. Int J Obes (Lond) 34: 1227-1229, 2010.

27. Xie Y, Shi X, Sheng K, Han G, Li W, Zhao Q, Jiang B, Feng J, Li J and Gu Y: PI3K/Akt signaling transduction pathway, erythropoiesis and glycolysis in hypoxia (Review). Mol Med Rep 19: 783-791, 2019.

28. Liu Y, Li W, Liu H, Peng Y, Yang Q, Xiao L, Liu Y and Liu F: Inhibition effect of small interfering RNA of connective tissue growth factor on the expression of extracellular matrix molecules in cultured human renal proximal tubular cells. Ren Fail 36: 278-284, 2014.

29. Qin D, Zhang GM, Xu X and Wang LY: The PI3K/Akt signaling pathway mediates the high glucose-induced expression of extracellular matrix molecules in human retinal pigment epithelial cells. J Diabetes Res 2015: 920280, 2015.

30. Chung EJ, Sohn YH, Kwon SH, Jung SA and Lee JH: Lithium chloride inhibits TGF- $\beta 1$-induced myofibroblast transdifferentiation via PI3K/Akt pathway in cultured fibroblasts from Tenon's capsule of the human eye. Biotechnol Lett 36: 1217-1224, 2014.

31. Guo X, Yang Y, Liu L, Liu X, Xu J, Wu K and Yu M: Pirfenidone induces G1 arrest in human Tenon's fibroblasts in vitro involving AKT and MAPK signaling pathways. J Ocul Pharmacol Ther 33 366-374, 2017.

32. Carducci A, Scafetta G, Siciliano C, Carnevale R, Rosa P, Coccia A, Mangino G, Bordin A, Vingolo EM, Pierelli L, et al: GMP-grade platelet lysate enhances proliferation and migration of tenon fibroblasts. Front Biosci (Elite Ed) 8: 84-99, 2016.

33. Li N, Cui J, Duan X, Chen H and Fan F: Suppression of type I collagen expression by miR-29b via PI3K, Akt, and Sp1 pathway in human Tenon's fibroblasts. Invest Ophthalmol Vis Sci 53: 1670-1678, 2012.

34. Zhang YE: Non-Smad pathways in TGF-beta signaling. Cell Res 19: 128-139, 2009.

35. Wen J, Lin X, Gao W, Qu B, Ling Y, Liu R and Yu M: MEK inhibition prevents TGF- $\beta 1$-induced myofibroblast trans differentiation in human tenon fibroblasts. Mol Med Rep 19: 468-476, 2019.

36. Addicks EM, Quigley HA, Green WR and Robin AL: Histologic characteristics of filtering blebs in glaucomatous eyes. Arch Ophthalmol 101: 795-798, 1983.

37. Hitchings RA and Grierson I: Clinico pathological correlation in eyes with failed fistulizing surgery. Trans Ophthalmol Soc U K 103: 84-88, 1983.

38. Mielke C, Dawda VK and Anand N: Intraoperative 5-fluorouracil application during primary trabeculectomy in Nigeria: A comparative study. Eye (Lond) 17: 829-834, 2003.

39. Wong TT, Khaw PT, Aung T, Foster PJ, Htoon HM, Oen FT, Gazzard G, Husain R, Devereux JG, Minassian D, et al: The singapore 5-fluorouracil trabeculectomy study: Effects on intraocular pressure control and disease progression at 3 years. Ophthalmology 116: 175-184, 2009.

40. Shin DH, Ren J, Juzych MS, Hughes BA, Kim C, Song MS, Yang KJ and Glover KB: Primary glaucoma triple procedure in patients with primary open-angle glaucoma: The effect of mitomycin-C in patients with and without prognostic factors for filtration failure. Am J Ophthalmol 125: 346-352, 1998.

41. Perkins TW, Gangnon R, Ladd W, Kaufman PL and Heatley GA: Trabeculectomy with mitomycin C: Intermediate-term results. J Glaucoma 7: 230-236, 1998. 